\title{
Overlapped Stenting for Treatment of an Extracranial Carotid Artery Aneurysm
}

\author{
Kiyoshi Tsuji, Norihito Fukawa, Nobuhiro Nakagawa, Tomonari Yabuuchi, Naoki Nakano, and Amami Kato
}

Extracranial carotid artery aneurysms (ECAAs) are rare, and their treatment is very difficult and challenging. The case of a 59-year-old woman who presented with a pulsatile mass on the left side of her neck, was diagnosed with a left-sided giant thrombosed common carotid artery aneurysm, and underwent definitive treatment by overlapped stenting, is presented. Overlapped stenting is a safe and an effective treatment procedure for ECAA. A covered stent would theoretically be reasonable and immediately effective, but its long-term patency remains unknown. Therefore, in patients lacking ischemic tolerance and those not requiring urgent intervention, overlapped stenting using uncovered stents is preferable.

Keywords: endovascular treatment, extracranial carotid artery aneurysm, overlapped stenting, uncovered stent

\section{Introduction}

Extracranial carotid artery aneurysms (ECAAs) are rare, but they are important for stroke physicians to recognize because of their high rate of cerebral embolism. ${ }^{1)}$ Open surgery is a common treatment and reported to be effective. ${ }^{2,3)}$ However, with recent advances in medical devices, endovascular treatment of ECAA is increasing, with covered stents being used in a relatively large number of cases. ${ }^{4} \mathrm{~A}$ patient who underwent overlapped stenting with uncovered stents for treatment of a giant thrombosed common carotid artery aneurysm is reported.

\section{Case Report}

A 59-year-old woman who was on hemodialysis for chronic renal failure presented to our hospital with chief complaint of a pulsatile mass on the left side of her neck. She had no recent history of head and neck surgery or trauma. Clinical examination revealed no clearly abnormal neurological findings. Three-dimensional computed tomography angiography performed for detailed examination revealed severe atherosclerosis with calcification from the aortic arch to the carotid arteries, and in the left common carotid artery (CCA), there was a giant thrombosed aneurysm measuring $5.3 \mathrm{~cm} \times 4.3 \mathrm{~cm}$ (Fig. 1A, B). In addition, there was asymptomatic near occlusion of the contralateral right internal carotid artery (ICA) (Fig. 1B).

Department of Neurosurgery, Kinki University School of Medicine, Osaka-Sayama, Osaka

Received: December 11, 2014; Accepted: February 13, 2015
Her condition, the purpose of treatment, the methods, and the risks involved were explained to the patient and her family, and her informed consent was obtained. Before treatment of the left CCA giant thrombosed aneurysm, a balloon occlusion test of the left ICA was performed. Since the patient immediately developed ischemic symptoms after balloon occlusion, the patient was deemed to lack ischemic
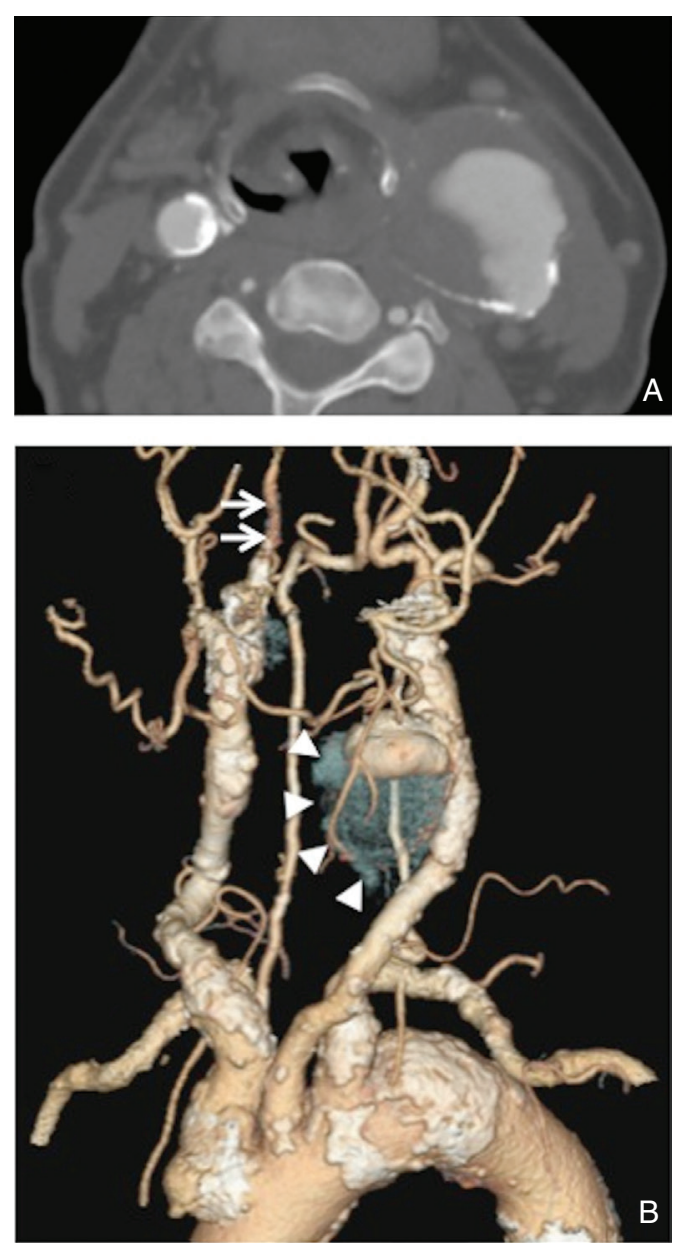

Fig. 1 A: Axial source image of three-dimensional computed tomography angiography (3D-CTA) showing a giant thrombosed aneurysm of the left common carotid artery (CCA). B: Volume-rendered 3D-CTA image (left anterior oblique $30^{\circ}$ view) showing severe calcification of the carotid arteries bilaterally and a giant thrombosed aneurysm in the left CCA. There is also near occlusion of the right internal carotid artery (ICA). Arrow: near occlusion of the right ICA, arrowhead: thrombosed area of the aneurysm. 

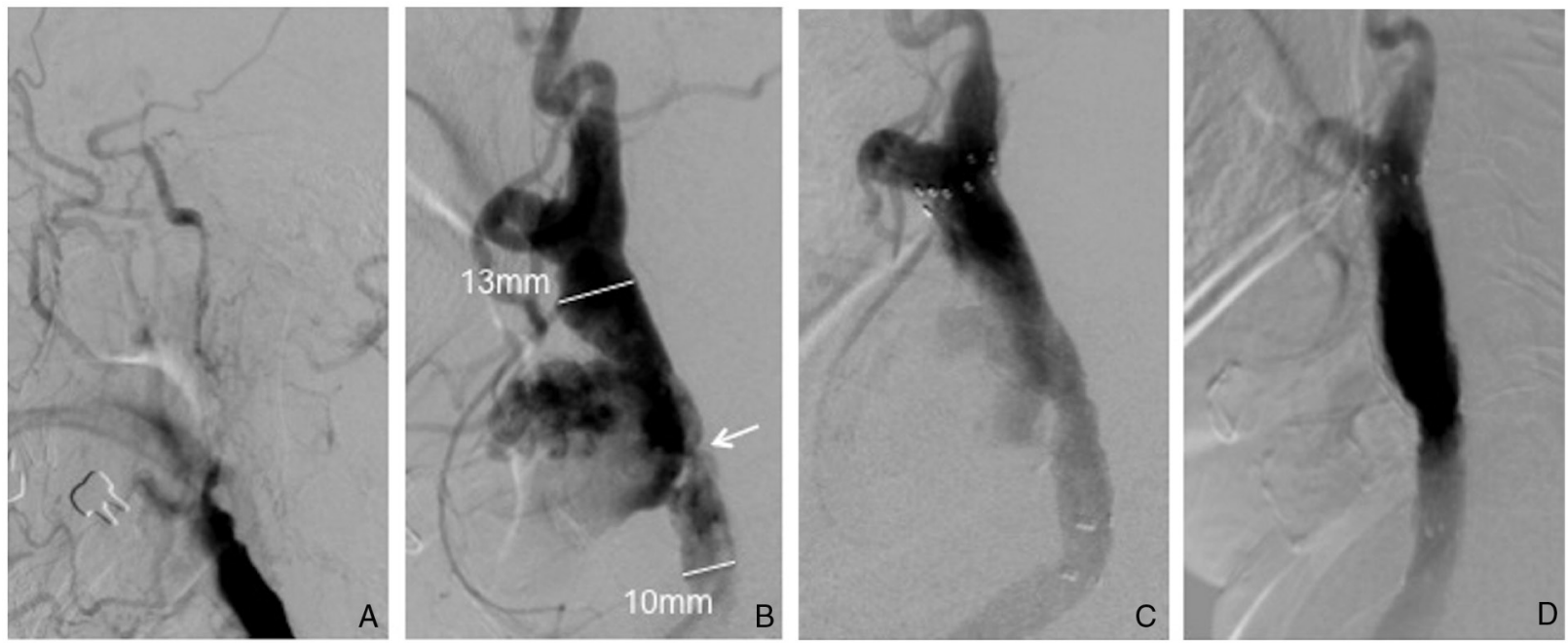

Fig. 2 A: Right carotid angiogram (lateral view) shows near occlusion of the right internal carotid artery (ICA). B: Left carotid angiogram (lateral view) before treatment. Because of severe atherosclerosis involving the origin of the left ICA and the left common carotid artery, there is ectatic dilation and severe stenosis proximal to the aneurysm (arrow: stenotic site). C: Left carotid angiogram (lateral view) just after placement of the two stents. Blood flow to the aneurysm has markedly decreased, and the stenotic site seen proximal to the aneurysm is now sufficiently dilated. D: Left carotid angiogram (lateral view) at follow-up 6 months after treatment. There is absolutely no blood flow to the aneurysm.

tolerance. For this reason, carotid artery stenting with the proximal balloon protection method for the near occlusion of the right ICA was attempted first (Fig. 2A). However, since the guidewire could not be passed through the lesion, the procedure was abandoned.

Treatment of the left CCA giant thrombosed aneurysm was rescheduled and performed another day. Under local anesthesia, with a transfemoral approach, a Launcher $8 \mathrm{~F}$ guiding catheter (Medtronic, Santa Rosa, California, USA) was guided to the left CCA. During the procedure, heparin was injected intravenously to control the activated clotting time to about $300 \mathrm{sec}$. The FilterWire EZ (Boston Scientific Corp., Natick, Massachusetts, USA) was guided to and placed in the petrous portion of the left ICA. Because of severe stenosis proximal to the aneurysm (Fig. 2B), this stenotic site was first predilated with an $8 \mathrm{~mm} \times 20 \mathrm{~mm}$ Sterling balloon (Boston Scientific). Since the maximum diameter of the left CCA was $13 \mathrm{~mm}$, a self-expanding $14 \mathrm{~mm} \times 60 \mathrm{~mm}$ E-Luminexx stent (BARD, Tempe, Arizona, USA) was placed to properly cover the aneurysm neck. The E-Luminexx stent is a 0.035 -inch compatible stent, and it was guided over two wires, a 0.014-inch CHIKAI microguidewire (Asahi Intecc, Aichi) and a 0.014inch FilterWire EZ. After post-dilation with a $9 \mathrm{~mm} \times 20 \mathrm{~mm}$ Sterling balloon, another $14 \mathrm{~mm} \times 60 \mathrm{~mm}$ E-Luminexx stent was placed at the same site. After overlapped stenting, blood flow to the aneurysm decreased markedly (Fig. 2C).

The pulsatile mass on the left side of her neck regressed gradually for 2 weeks post-procedure. Carotid ultrasound 1 month post-procedure showed complete thrombosis of the aneurysm and reduction of the aneurysm size compared with pre-procedure (Fig. 3A, B). At 2 months post-procedure, the pulsatile mass on the left side of her neck had almost disappeared. Follow-up angiography 6 months post-procedure showed absolutely no blood flow to the aneurysm (Fig. 2D). Subsequently, the patient had no cerebral ischemic
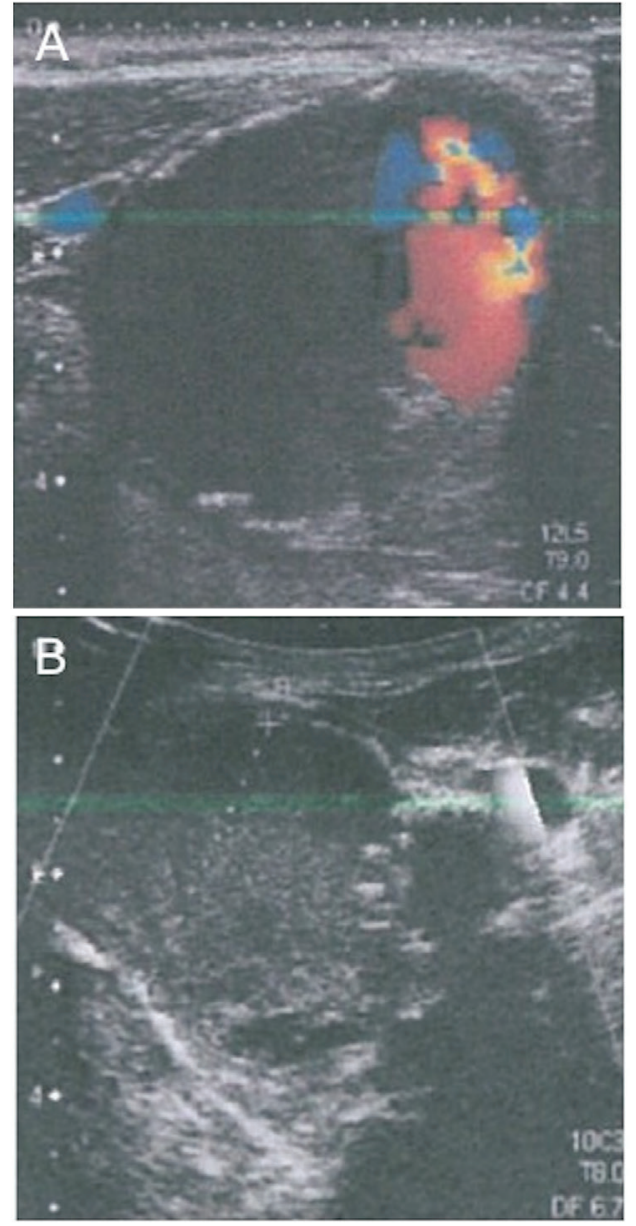

Fig. 3 A, B: Carotid ultrasound before (A) and 1 month after (B) endovascular treatment. There is complete thrombosis of the aneurysm in the carotid ultrasound 1 month post-procedure, and the aneurysm size appears smaller than before the procedure. 
complications and was doing well at outpatient follow-up. Unfortunately, at 16 months post-procedure, she had a sudden acute myocardial infarction and died.

\section{Discussion}

ECAAs are rare, accounting for only about $0.4-4 \%$ of all peripheral artery aneurysms. ${ }^{4)}$ The causes of ECAA are reported to include atherosclerosis, trauma, infection, postcarotid endarterectomy, fibromuscular dysplasia, and Takayasu disease; however, atherosclerosis is the most common cause, occurring in about $70 \%$ of cases. ${ }^{3,5}$ Since the present patient was on dialysis for chronic renal failure, and there was no history of trauma or infection, atherosclerosis was the likely cause.

The most common mode of presentation of an ECAA is cerebral ischemic symptoms due to an embolism from an intra-aneurysmal thrombus, and about one-half of patients has a transient ischemic attack or cerebral infarction. ${ }^{1,3,4)}$ Other modes of presentation include a pulsatile mass in the neck, as in the present patient, as well as lower cranial nerve palsies and Horner's syndrome. When symptoms of compression due to an aneurysm are severe, airway obstruction may develop, but aneurysm rupture is relatively uncommon.

When an ECAA is managed conservatively without surgical intervention, high rates of cerebral infarction during follow-up have been reported. ${ }^{1)}$ Therefore, aggressive treatment intervention is necessary. Open surgery is regarded as standard treatment, and carotid artery ligation, aneurysmectomy with patch angioplasty, aneurysmectomy with endto-end anastomosis, and aneurysmectomy with interposition bypass grafting have been performed. ${ }^{2,3,5)}$ Carotid artery ligation is technically easy, but in a patient like the present one without ischemic tolerance and with a lesion involving the CCA, this cannot be performed. Moreover, surgical procedures other than carotid artery ligation are technically more difficult, and the risk of lower cranial nerve palsies after the procedure is higher. ${ }^{3)}$

Endovascular treatment is less invasive than open surgery, and endovascular treatment for ECAA is increasingly being reported in recent years. ${ }^{6)}$ However, covered stents are used relatively often in such cases. ${ }^{4)}$ Although a covered stent would certainly be reasonable, rapidly effective, and seem to be an ideal device, the long-term patency of covered stents is unknown. Covering materials for covered stents include expanded polytetrafluoroethylene (ePTFE), polyethylene terephthalate, and silicone. Of these, ePTFE induces few foreign-body reactions, ${ }^{7}$ so it is widely used in vascular procedures. However, it has been reported that the primary patency rate of ePTFE-covered stents at 12 months after placement in the femoropopliteal artery was approximately $55 \% .^{8)}$ Therefore, in a patient like the present one without ischemic tolerance, a covered stent should not be used. For this reason, overlapped stenting using open-cell type E-Luminexx uncovered stents was performed.

Overlapped stenting, by virtue of increased stent mesh density, is likely to enhance the flow diversion effect and promote intra-aneurysmal thrombosis. ${ }^{9-11)}$ The significance of increased stent mesh density might seem to favor a closedcell type stent, but closed-cell type stents are characterized by poorer conformability to the vessel wall. The present patient had severe atherosclerosis of the CCA, with irregularities of the vessel wall and differences in luminal diameter, and moreover, there was severe stenosis proximal to the aneurysm. If a closed-cell type stent were placed in this type of situation, the stent would not conform well to the vessel wall at the aneurysm neck site, so space might develop between the stent and aneurysm neck, thus reducing the flow diversion effect. Therefore, an open-cell type stent with better stent conformability was selected. Although an opencell type stent has less stent mesh density, a satisfactory flow diversion effect was achieved in the present patient by overlapped stenting.

Endovascular treatment of ECAAs may also include a method of combining coil packing into the aneurysm sac with stent placement. ${ }^{12-15)}$ This is effective for small aneurysms, but for large aneurysms, many coils are needed, and the coils themselves can cause a mass effect, so they are not recommended.

Overlapped stenting provided definitive treatment for the ECAA in the present patient, but this technique may not be effective in all such cases. Therefore, treatment options must be carefully considered in each patient based on that patient's symptoms, ischemic tolerance, and the cause, shape, and size of the aneurysm. ${ }^{14)}$ Debate continues on whether to use covered stents or uncovered stents. However, the long-term patency of covered stents is unknown. Therefore, in patients without ischemic tolerance, and in those who are not in an urgent situation, such as aneurysm rupture or airway obstruction, use of an uncovered stent is preferable.

Finally, according to the literature, cardiac complications are common in patients with an ECAA. Zhou et al. ${ }^{6}$ reported that, among 42 patients who were followed up for a mean of 4.6 years after ECAA treatment, 11 patients died due to cardiac disease. This is convincing, considering that atherosclerosis is the most common cause of ECAA. When treating patients with an atherosclerotic ECAA, referral to a cardiologist for a consultation is recommended.

\section{Conclusion}

Overlapped stenting is a safe and effective treatment procedure for an ECAA. In patients without ischemic tolerance, and in those not requiring urgent intervention, overlapped stenting with uncovered stents instead of a covered stent may be preferable. Since patients with an atherosclerotic ECAA are also likely to have underlying cardiac disease, they should be referred to a cardiologist for a consultation.

\section{Conflicts of Interest Disclosure}

The authors declare that they have no conflicts of interest. All authors who are members of The Japan Neurosurgical Society (JNS) have registered online Self-reported COI Disclosure Statement Forms through the website for JNS members. 


\section{References}

1) Zwolak RM, Whitehouse WM, Knake JE, Bernfeld BD, Zelenock GB, Cronenwett JL, Erlandson EE, Kazmers A, Graham LM, Lindenauer SM: Atherosclerotic extracranial carotid artery aneurysms. J Vasc Surg 1: 415-422, 1984

2) Rosset E, Albertini JN, Magnan PE, Ede B, Thomassin JM, Branchereau A: Surgical treatment of extracranial internal carotid artery aneurysms. J Vasc Surg 31: 713-723, 2000

3) Attigah N, Külkens S, Zausig N, Hansmann J, Ringleb P, Hakimi M, Eckstein HH, Allenberg JR, Böckler D: Surgical therapy of extracranial carotid artery aneurysms: long-term results over a 24-year period. Eur J Vasc Endovasc Surg 37: 127-133, 2009

4) Li Z, Chang G, Yao C, Guo L, Liu Y, Wang M, Liu D, Wang S: Endovascular stenting of extracranial carotid artery aneurysm: a systematic review. Eur J Vasc Endovasc Surg 42: 419-426, 2011

5) Angiletta D, Pulli R, Marinazzo D, Frotino P, Maiellaro L, Regina G: Surgical and endovascular treatment of extracranial carotid artery aneurysms: early and long-term results of a single center. Ann Vasc Surg 28: 659-664, 2014

6) Zhou W, Lin PH, Bush RL, Peden E, Guerrero MA, Terramani T, Lubbe DF, Nguyen L, Lumsden AB: Carotid artery aneurysm: evolution of management over two decades. J Vasc Surg 43: 493-496; discussion 497, 2006

7) Cejna M, Virmani R, Jones R, Bergmeister H, Losert U, Xu Z, Yang P, Schoder M, Lammer J: Biocompatibility and performance of the Wallstent and several covered stents in a sheep iliac artery model. $J$ Vasc Interv Radiol 12: 351-358, 2001

8) Geraghty PJ, Mewissen MW, Jaff MR, Ansel GM; VIBRANT Investigators: Three-year results of the VIBRANT trial of VIABAHN endoprosthesis versus bare nitinol stent implantation for complex superficial femoral artery occlusive disease. J Vasc Surg 58: 386-395. e4, 2013

9) Pan YH, Lin Y, Ding SH, Chen L, Liang YM, Yin YH, Bao YH, Gao GY, Qiu YM, Jiang JY: Endovascular treatment of the extracrania carotid pseudoaneurysms resulting from stab penetrating injury using overlapping bare stents. Vasc Endovascular Surg 48: 337-341, 2014

10) Benndorf G, Wellnhofer E, Schneider GH: Doubled stenting for effective occlusion of a dissecting carotid artery aneurysm. Interv Neuroradiol 6: 343-348, 2000

11) Benndorf G, Herbon U, Sollmann WP, Campi A: Treatment of a ruptured dissecting vertebral artery aneurysm with double stent placement: case report. AJNR Am J Neuroradiol 22: 1844-1848, 2001

12) Klein GE, Szolar DH, Raith J, Frühwirth H, Pascher O, Hausegger KA: Posttraumatic extracranial aneurysm of the internal carotid artery: combined endovascular treatment with coils and stents. AJNR Am J Neuroradiol 18: 1261-1264, 1997

13) Perez-Cruet MJ, Patwardhan RV, Mawad ME, Rose JE: Treatment of dissecting pseudoaneurysm of the cervical internal carotid artery using a wall stent and detachable coils: case report. Neurosurgery 40: 622-625; discussion 625-626, 1997

14) Taha MM, Nakahara I, Higashi T, Iwamuro $Y$, Watanabe $Y$, Taki $W$ : Interventional neuroradiological techniques for the treatment of aneurysms of the supra-aortic extracranial arteries. Neurol Med Chir (Tokyo) 50: 275-280, 2010

15) Liu AY, Paulsen RD, Marcellus ML, Steinberg GK, Marks MP: Long-term outcomes after carotid stent placement treatment of carotid artery dissection. Neurosurgery 45: 1368-1373; discussion 1373-1374, 1999

Corresponding author:

Kiyoshi Tsuji, MD, PhD, Department of Neurosurgery, Kinki University School of Medicine, 377-2 Ohno-Higashi, Osaka-Sayama, Osaka 589-8511, Japan.

$\triangle$ kiyoshi-tsuji@med.kindai.ac.jp 